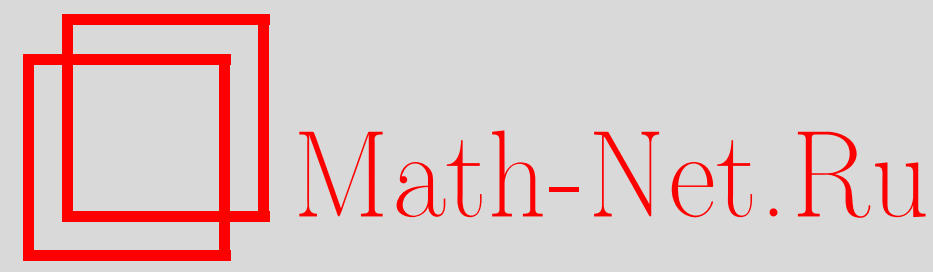

К. Г. Малютин, Ряды Фурье и $\delta$-субгармонические функции конечного $\gamma$-типа в полуплоскости, Матем. сб., 2001, том 192, номер 6, 51-70

DOI: https://doi.org/10.4213/sm572

Использование Общероссийского математического портала Math-Net.Ru подразумевает, что вы прочитали и согласны с пользовательским соглашением http://www.mathnet.ru/rus/agreement

Параметры загрузки:

IP : 52.205 .19 .152

26 апреля 2023 г., $14: 40: 15$ 


\section{К.Г. Малютин \\ Ряды Фурье и $\delta$-субгармонические функции конечного $\gamma$-типа в полуплоскости}

Пусть $\gamma(r)$ - функция роста. Пусть $v(z)$ является истинно $\delta$-субгармонической функцией в смысле Гришина в комплексной полуплоскости, т.е. $v=v_{1}-v_{2}$, где $v_{1}, v_{2}$ - истинно субгармонические функции $\left(\lim _{\sup _{z \rightarrow t}} v_{i}(z) \leqslant 0, i=\right.$ 1,2 , для каждого вешественного $t$ ), пусть $\lambda=\lambda_{+}-\lambda_{-}-$ее полная мера, и пусть $T(r, v)$ - ее неванлинновская характеристика. Класс $J \delta(\gamma)$ функций конечного $\gamma$-типа определяется следуюшим образом: $v \in J \delta(\gamma)$, если существуют положительњые константы $A$ и $B$ такие, что $T(r, v) \leqslant A \gamma(B r) / r$. Коэфофициенты Фурье функции $v$ определяются стандартным способом: $c_{k}(r, v)=$ $\frac{2}{\pi} \int_{0}^{\pi} v\left(r e^{i \theta}\right) \sin k \theta d \theta, r>0, k \in \mathbb{N}$. Основной результат работы заключается в эквивалентности следующих утверждений: 1) $v \in J \delta(\gamma)$; 2) $N(r) \leqslant$ $A_{1} \gamma\left(B_{1} r\right) / r$, где $N(r)=N\left(r, \lambda_{+}\right)$или $N(r)=N\left(r, \lambda_{-}\right),\left|c_{k}(r, v)\right| \leqslant A_{2} \gamma\left(B_{2} r\right)$. Кроме того, доказывается, что $J \delta(\gamma)=J S(\gamma)-J S(\gamma)$, где $J S(\gamma)$ - класс истинно субгармонических функций конечного $\gamma$-типа.

Библиографоия: 15 названий.

Введение. В 60-х годах в работах американских математиков Л. Рубела, Б. Тейлора, Д. Майлза, Д. Шиа и др. начал широко применяться метод рядов Фурье для изучения свойств целых и мероморфных функций. Этот метод является эффективным при решении ряда обших задач теории мероморфных функций и устанавливает ее связь с теорией рядов Фурье. Одним из преимуществ этого метода является то, что он позволяет исследовать функции с довольно нерегулярным ростом на бесконечности и функции бесконечного порядка.

Строго положительная, непрерывная, возрастающая и неограниченная функция $\gamma(r)$, определенная на $[0, \infty)$, назьвается функиией роста. Пусть $f$ - мероморфная в комплексной плоскости функция, $Z(f)(W(f))$ - множество ее нулей (полюсов), $T(r, f)$ - неванлинновская характеристика, $c_{k}(r, f)$ - коэффициенты Фурье функции $f$. Функция $f$ называется функиией конечного $\gamma$-типа, если существуют положительные постоянные $A$ и $B$ такие, что $T(r, f) \leqslant A \gamma(B r)$ для всех $r>0$. Класс таких функций обозначим через $\Gamma$, через $\Gamma_{E}$ обозначим класс целых функций конечного $\gamma$-типа. Ниже через $A, B, \ldots$ мы будем обозначать положительные константы, которые могут изменяться на протяжении статьи. Рубел и Тейлор [1] доказали эквивалентность трех следуюших утверждений:

1) $f \in \Gamma$;

2) последовательность $Z(f)$ (или $W(f))$ имеет конечную $\gamma$-плотность,

$$
c_{k}(r, f) \leqslant A \gamma(B r), \quad k \in \mathbb{Z},
$$


при некоторых положительных $A, B$ и всех $r>0$;

3 ) последовательности $Z(f)$ и $W(f)$ имеют конечную $\gamma$-плотность,

$$
\mathrm{c}_{k}(r, f) \leqslant \frac{A}{|k|+1} \gamma(B r), \quad k \in \mathbb{Z}
$$

при некоторых (возможно, других, но не зависящих от $k$ ) положительных $A, B$ и всех $r>0$.

Используя метод рядов Фурье, Д. Майлз [2] решил не поддававшуюся решению на протяжении ряда лет проблему представления мероморфной функции $f \in \Gamma$ в виде частного двух целых функций из класса $\Gamma_{E}: \Gamma=\Gamma_{E} / \Gamma_{E}$. В 80-е годы важные результаты в этом направлении были получены А. А. Кондратюком [3]-[5], которьй обобщил теорию Левина - Пфлюгера целых функций вполне регулярного роста на мероморфные функции произвольного $\gamma$-типа.

В данной работе мы распространяем некоторые из отмеченных выше результатов на функции, определенные в полуплоскости. Причем мы доказываем наши утверждения сразу для $\delta$-субгармонических функций. Переход в полуплоскость вызывает определенные трудности, связанные со сложным поведением функции в окрестности границы. Отличие от плоскости проявляется уже при получении критериев принадлежности $\delta$-субгармонической функции заданному классу. Так, например, никакое обобщение аналога пункта 3) критерия Рубела-Тейлора в полуплоскости невозможно.

Автор выражает глубокую признательность А.Ф. Гришину за ценные замечания, сделанные в процессе работы над этой статьей.

1. Основные результаты. Обозначим через $J \delta$ класс истинно $\delta$-субгармонических функций, а через $J \delta(\gamma(r))$ - класс истинно $\delta$-субгармонических функций конечного $\gamma$-типа (определения этих классов даны ниже) в верхней полуплоскости.

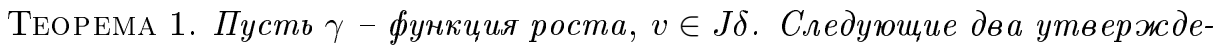
ния әквивалентнь:

1) $v \in J \delta(\gamma(r))$

2) мера $\lambda_{+}(v)\left(\right.$ или $\left.\lambda_{-}(v)\right)$ имеет конечную $\gamma-$ плотность,

$$
\left|c_{k}(r, v)\right| \leqslant A \gamma(B r), \quad k \in \mathbb{N}
$$

при некоторых положительных $A, B$ и всех $r>0$.

Здесь $\lambda(v)=\lambda_{+}(v)-\lambda_{-}(v)-$ полная мера функции $v$.

Заметим, что из условия 1$)$ не следует такое усиление 3$):\left|c_{k}(r)\right| \leqslant \varepsilon_{k} \gamma(B r)$, где $\varepsilon_{k} \rightarrow 0$ при $k \rightarrow \infty$.

Справедлив также аналог результата Д. Майлза для полуплоскости: $J \delta(\gamma(r))=$ $J S(\gamma(r))-J S(\gamma(r))$, где $J S(\gamma(r))$ - класс истинно субгармонических функций конечного $\gamma$-типа. 
2. Классы функций в $\mathbb{C}_{+} \cdot$ В работе будем использовать терминологию из [6], [7]. Обозначим через $\mathbb{C}_{+}=\{z: \operatorname{Im} z>0\}$ верхнюю полуплоскость. Через $C(a, r)$ будем обозначать открытый круг радиуса $r$ с центром в точке $a$, через $\Omega_{+}-$пересечение множества $\Omega$ с полуплоскостью $\mathbb{C}_{+}: \Omega_{+}=\Omega \cap \mathbb{C}_{+}$. Субгармоническая в $\mathbb{C}_{+}$функция $v$ назьвается истинно субгармонической, если $\overline{\lim }_{z \rightarrow t} v(z) \leqslant 0$ для любого числа $t \in \mathbb{R}$. Класс истинно субгармонических функций в $\mathbb{C}_{+}$обозначим через $J S$. Пусть $S K-$ класс субгармонических функций в $\mathbb{C}_{+}$, имеющих положительную гармоническую мажоранту в любой ограниченной области в $\mathbb{C}_{+}$. Функции класса $S K$ обладают следующими свойствами [6]:

а) $v(z)$ имеет некасательный предел $v(t)$ почти всюду на вещественной оси, $v(t) \in L_{\mathrm{loc}}^{1}(-\infty, \infty)$

b) на вещественной прямой сушествует знакопеременная мера $\nu$ такая, что

$$
\lim _{y \rightarrow+0} \int_{a}^{b} v(t+i y) d t=\nu([a, b])-\frac{1}{2} \nu(\{a\})-\frac{1}{2} \nu(\{b\}) .
$$

Мера $\nu$ называется граничной мерой функции $v$;

c) $d \nu(t)=v(t) d t+d \sigma(t)$, где $\sigma-$ сингулярная мера относительно меры Лебега.

Для функции $v \in S K$ определим, следуя [6], полную меру $\lambda$ как

$$
\lambda(K)=2 \pi \int_{\mathbb{C}_{+} \cap K} \operatorname{Im} \zeta d \mu(\zeta)-\nu(K),
$$

где $\mu$ - риссовская мера функции $v$. Мера $\lambda$ обладает следующими свойствами:

1) $\lambda$-конечная мера на каждом компакте $K \subset \mathbb{C}$;

2) $\lambda$ - положительная мера вне $\mathbb{R}$;

3) $\lambda$ равна нулю в полуплоскости $\mathbb{C}_{-}=\{z: \operatorname{Im} z<0\}$.

Наоборот, если мера $\lambda$ удовлетворяет условиям 1)-3), то существует функция $v \in S K$ с полной мерой, равной $\lambda$. Совокупность условий 1)-3) в дальнейшем будем обозначать через $\{\mathbf{G}\}$; если, кроме того, мера $\lambda$ неотрицательная и на $\mathbb{R}$, то через $\left\{\mathbf{G}^{+}\right\}$.

Если $D$ - ограниченная область в $\mathbb{C}_{+}, D_{1}=D \cup(\partial D \cap \mathbb{R}), v \in S K, z \in D$, то

$$
v(z)=\frac{1}{2 \pi} \iint_{D_{1}} \frac{1}{\operatorname{Im} \zeta} \ln \left|\frac{z-\zeta}{z-\bar{\zeta}}\right| d \lambda(\zeta)+h(z)
$$

где $h$ - гармоническая функция в $D$, а если $[a, b] \subset\{\mathbb{R} \cap \partial D\}$, то $h$ допускает непрерывное продолжение нулем на $(a, b), \frac{1}{\operatorname{Im} \zeta} \ln \left|\frac{z-\zeta}{z-\bar{\zeta}}\right|$ считается продолженной по непрерывности на вещественную ось. Полная мера $\lambda$ определяет функцию $v \in S K$ так же, как мера Рисса $\mu$ определяет субгармоническую функцию в $\mathbb{C}$. Точнее, если функции $v_{1}, v_{2} \in S K$ и каждая имеет полную меру $\lambda$, то существует вешественная целая функция $g$ такая, что $v_{2}(z)-v_{1}(z)=\operatorname{Im} g(z), z \in \mathbb{C}_{+}$.

Справедливы следующие утверждения [6]. 
УТВЕРЖДЕНИЕ 2.1. $J S \subset S K$.

Полная мера функции $v \in J S$ является положительной мерой, чем и обоснован термин "истинно субгармоническая функция". Отметим также, что множество $J S$ является конусом, т.е. если $v_{1}, v_{2} \in J S, \alpha \geqslant 0$, то $v_{1}+v_{2}, \alpha v_{1} \in J S$.

Введем теперь класс истинно $\delta$-субгармонических функций $J \delta=J S-J S$. Заметим, что $J \delta$ - есть наиболее широкий класс $\delta$-субгармонических функций в полуплоскости, для которых можно определить неванлинновскую характеристику. Действительно, все неванлинновские характеристики $\delta$-субгармонической функции определяются посредством меры, соответствующей этой функции. Так, для функции, $\delta$-субгармонической во всей плоскости, такой мерой будет ее риссовская мера, для функции класса $J \delta$ - ее полная мера, в определение которой входит граничная мера $\nu$. Граничное поведение любой функции, на входящей в класс $J \delta$, определяется обязательно некоторой обобщенной функцией на вешественной оси, не являюшейся мерой.

УТВЕРЖ ДЕНИЕ 2.2. $J \delta=S K-S K$.

Для заданной меры $\lambda$ обозначим

$$
d \lambda_{m}(\zeta)=\frac{\sin m \varphi}{\sin \varphi} \tau^{m-1} d \lambda(\zeta) \quad\left(\zeta=\tau e^{i \varphi}\right), \quad \lambda_{m}(r)=\lambda_{m}(\overline{C(0, r)}),
$$

где $\frac{\sin m \varphi}{\sin \varphi}$ при $\varphi=0, \pi$ определяется по непрерьвности.

Следуюшая формула - это формула Карлемана в обозначениях А.. . Гришина:

$$
\frac{1}{r^{k}} \int_{0}^{\pi} v\left(r e^{i \varphi}\right) \sin k \varphi d \varphi=\int_{r_{0}}^{r} \frac{\lambda_{k}(t)}{t^{2 k+1}} d t+\frac{1}{r_{0}^{k}} \int_{0}^{\pi} v\left(r_{0} e^{i \varphi}\right) \sin k \varphi d \varphi
$$

в частности, при $k=1$

$$
\frac{1}{r} \int_{0}^{\pi} v\left(r e^{i \varphi}\right) \sin \varphi d \varphi=\int_{r_{0}}^{r} \frac{\lambda(t)}{t^{3}} d t+\frac{1}{r_{0}} \int_{0}^{\pi} v\left(r_{0} e^{i \varphi}\right) \sin \varphi d \varphi
$$

для всех $r>r_{0}$.

Отметим еше неравенство, которое будет полезно в дальнейшем:

$$
\begin{aligned}
\left|\lambda_{m}(r)\right| & =\left|\iint_{\overline{C(0, r)}} d \lambda_{m}(\zeta)\right|=\left|\iint_{\overline{C(0, r)}} \frac{\sin m \varphi}{\sin \varphi} \tau^{m-1} d \lambda(\zeta)\right| \\
& \leqslant m \iint_{\overline{C(0, r)}} \tau^{m-1} d|\lambda|(\zeta) \leqslant m r^{m-1}|\lambda|(r) .
\end{aligned}
$$

Обозначим через $D_{+}\left(R_{1}, R_{2}\right)=\overline{C_{+}\left(0, R_{2}\right) \backslash C_{+}\left(0, R_{1}\right)}, R_{1}<R_{2}$. Для функций $v \in J \delta$ справедливо представление в полукольце $z \in D_{+}\left(R_{1}, R_{2}\right)$ :

$$
\begin{aligned}
v(z)= & -\frac{1}{2 \pi} \iint_{D_{+}\left(R_{1}, R_{2}\right)} K(z, \zeta) d \lambda(\zeta)+\frac{R_{2}}{2 \pi} \int_{0}^{\pi} \frac{\partial G\left(z, R_{2} e^{i \varphi}\right)}{\partial n} v\left(R_{2} e^{i \varphi}\right) d \varphi \\
& +\frac{R_{1}}{2 \pi} \int_{0}^{\pi} \frac{\partial G\left(z, R_{1} e^{i \varphi}\right)}{\partial n} v\left(R_{1} e^{i \varphi}\right) d \varphi
\end{aligned}
$$


и в полукруге $z \in C_{+}(0, R)$ :

$$
v(z)=-\frac{1}{2 \pi} \iint_{C_{+}(0, R)} K(z, \zeta) d \lambda(\zeta)+\frac{R}{2 \pi} \int_{0}^{\pi} \frac{\partial G\left(z, R e^{i \varphi}\right)}{\partial n} v\left(R e^{i \varphi}\right) d \varphi
$$

где $G(z, \zeta)$ - функция Грина полукольца (полукруга), $\frac{\partial G}{\partial n}$ означает производную по внутренней нормали, функция $K(z, \zeta)=\frac{1}{\operatorname{Im} \zeta} G(z, \zeta)$ при $\zeta \in D_{+}\left(R_{1}, R_{2}\right)(z \in$ $\left.C_{+}(0, R)\right)$ продолжена по непрерывности на вешественную ось при $R_{1} \leqslant|t| \leqslant R_{2}$.

Используя теорию эллиптических функций (см., например, [8; гл. VIII]), можно получить разложения ядра в формуле (4) при $R_{1}=q R, R_{2}=R / q, q \in(0,1)$, $z=r e^{i \theta}, \zeta=\tau e^{i \varphi}$

$$
\begin{aligned}
& G(z, \zeta)=2 \sum_{m=1}^{\infty} \frac{1}{m\left(1-q^{4 m}\right)}\left(\frac{\tau}{r}\right)^{m}\left(1-\frac{q^{2 m} r^{2 m}}{R^{2 m}}\right)\left(1-\frac{q^{2 m} R^{2 m}}{\tau^{2 m}}\right) \\
& \times \sin m \theta \sin m \varphi, \quad q R \leqslant \tau<r<\frac{1}{q} R, \\
& G(z, \zeta)=2 \sum_{m=1}^{\infty} \frac{1}{m\left(1-q^{4 m}\right)}\left(\frac{r}{\tau}\right)^{m}\left(1-\frac{q^{2 m} R^{2 m}}{r^{2 m}}\right)\left(1-\frac{q^{2 m} \tau^{2 m}}{R^{2 m}}\right) \\
& \times \sin m \theta \sin m \varphi, \quad q R \leqslant r<\tau \leqslant \frac{1}{q} R, \\
& \frac{\partial G(z, t)}{\partial n}=\frac{2}{t} \sum_{m=1}^{\infty} \frac{1}{m\left(1-q^{4 m}\right)}\left(\frac{t}{r}\right)^{m}\left(1-\frac{q^{2 m} R^{2 m}}{t^{2 m}}\right) \\
& \times\left(1-\frac{q^{2 m} r^{2 m}}{R^{2 m}}\right) \sin m \theta, \quad q R \leqslant|t| \leqslant r \leqslant \frac{1}{q} R, \\
& \frac{\partial G(z, t)}{\partial n}=\frac{2}{t} \sum_{m=1}^{\infty} \frac{1}{m\left(1-q^{4 m}\right)}\left(\frac{r}{t}\right)^{m}\left(1-\frac{q^{2 m} t^{2 m}}{R^{2 m}}\right) \\
& \times\left(1-\frac{q^{2 m} R^{2 m}}{r^{2 m}}\right) \sin m \theta, \quad q R \leqslant r \leqslant|t| \leqslant \frac{1}{q} R, \\
& \frac{\partial G\left(z, q R e^{i \varphi}\right)}{\partial n}=\frac{4}{q R} \sum_{m=1}^{\infty} \frac{1}{1-q^{4 m}}\left(\frac{q R}{r}\right)^{m}\left(1-\frac{q^{2 m} r^{2 m}}{R^{2 m}}\right) \sin m \theta \sin m \varphi, \\
& \frac{\partial G\left(z,(1 / q) R e^{i \varphi}\right)}{\partial n}=\frac{4 q}{R} \sum_{m=1}^{\infty} \frac{1}{1-q^{4 m}}\left(\frac{q r}{R}\right)^{m}\left(1-\frac{q^{2 m} R^{2 m}}{r^{2 m}}\right) \sin m \theta \sin m \varphi \text {. }
\end{aligned}
$$


3. Коэффициенты Фурье функций класса $J \delta$. Коэффициенты Фурье функции $v \in J \delta$ определяются обычным образом [9]:

$$
c_{k}(r, v)=\frac{2}{\pi} \int_{0}^{\pi} v\left(r e^{i \theta}\right) \sin k \theta d \theta, \quad k \in \mathbb{N} .
$$

Из формулы (1) получим следующие выражения для коэффициентов Фурье при $r>r_{0}$ :

$$
c_{k}(r, v)=\alpha_{k} r^{k}+\frac{2 r^{k}}{\pi} \int_{r_{0}}^{r} \frac{\lambda_{k}(t)}{t^{2 k+1}} d t, \quad k \in \mathbb{N},
$$

где $\alpha_{k}=r_{0}^{-k} c_{k}\left(r_{0}, v\right)$.

Применяя формулу интегрирования по частям к интегралу в (11), получим

$$
\begin{aligned}
& c_{k}(r, v)=\alpha_{k} r^{k}+\frac{r^{k}}{\pi k r_{0}^{2 k}} \iint_{\overline{C_{+}\left(0, r_{0}\right)}} \frac{\sin k \varphi}{\operatorname{Im} \zeta} \tau^{k} d \lambda(\zeta) \\
& +\frac{r^{k}}{\pi k} \iint_{D_{+}\left(r_{0}, r\right)} \frac{\sin k \varphi}{\tau^{k} \operatorname{Im} \zeta} d \lambda(\zeta)-\frac{1}{r^{k} \pi k} \iint_{\overline{C_{+}(0, r)}} \frac{\sin k \varphi}{\operatorname{Im} \zeta} \tau^{k} d \lambda(\zeta),
\end{aligned}
$$

где $\zeta=\tau e^{i \varphi}$

УТВЕРЖДЕНИЕ 3.1. Коэффициенты Фурье $c_{k}(r, v)$ функции класса Јб являются непрерывными функциями от $r$.

Это вытекает из того, что правые части соотношений (11) являются непрерьвными в каждой точке $r$ функциями.

4. Суб- и $\delta$-субгармонические функции конечного $\gamma$-типа. Пусть $v \in$ $J \delta, v=v_{+}-v_{-}, \lambda-$ полная мера функции $v, \lambda=\lambda_{+}-\lambda_{-}-$жорданово разложение меры $\lambda$ (заметим, что $\lambda_{-}$не есть полная мера $v_{-}$). Обозначим через

$$
\begin{gathered}
m(r, v):=\frac{1}{r} \int_{0}^{\pi} v_{+}\left(r e^{i \varphi}\right) \sin \varphi d \varphi, \quad N(r, v):=\int_{r_{0}}^{r} \frac{\lambda_{-}(t)}{t^{3}} d t, \\
T(r, v):=m(r, v)+N(r, v)+m\left(r_{0},-v\right),
\end{gathered}
$$

где $r_{0}$ - произвольное положительное число, при желании можно считать $r_{0}=1$, $r>r_{0}$.

В этих обозначениях формула Карлемана (2)может быть записана в следующем виде:

$$
T(r, v)=T(r,-v) .
$$

Мы предположим теперь, что функция роста $\gamma$ удовлетворяет условию:

$$
\varliminf_{r \rightarrow \infty} \frac{\gamma(r)}{r}>0 .
$$

Функция $v \in J \delta$ назьвается функцией конечного $\gamma$-типа, если существуют постоянные $A$ и $B>0$ такие, что

$$
T(r, v) \leqslant \frac{A}{r} \gamma(B r)
$$

для всех $r>r_{0}$

Класс данных $\delta$-субгармонических функций конечного $\gamma$-типа обозначим через $J \delta(\gamma(r))$. Через $J S(\gamma(r))$ обозначим класс истинно субгармонических функций конечного $\gamma$-типа. 
ЛЕмма 1. Класс $J \delta(\gamma(r))$ - вещественное линейное пространство, а класс $J S(\gamma(r))-$ конус.

Это утверждение вытекает из (13) и неравенства $T\left(r, \sum v_{j}\right) \leqslant \sum T\left(r, v_{j}\right)$.

Положительная мера $\lambda$ имеет конечную $\gamma$-плотность, если при некоторых положительных $A$ и $B$ выполняется неравенство

$$
N(r, \lambda):=\int_{r_{0}}^{r} \frac{\lambda(t)}{t^{3}} d t \leqslant \frac{A}{r} \gamma(B r)
$$

для всех $r>r_{0}$.

Положительная мера $\lambda$ в комплексной плоскости называется мерой конечного $\gamma$-muna, если сушествуют положительные постоянные $A$ и $B$ такие, что для всех $r>0$

$$
\lambda(r) \leqslant r A \gamma(B r)
$$

ЛЕмма 2. Если мера $\lambda$ имеет конечную $\gamma$-плотность, то она является мерой конечного $\gamma$-типа.

Доказательством служат неравенства

$$
N(e r, \lambda)=\int_{r_{0}}^{e r} \frac{\lambda(t)}{t^{3}} d t \geqslant \int_{r}^{e r} \frac{\lambda(t)}{t^{3}} d t \geqslant \frac{\lambda(r)}{e^{2} r^{2}}
$$

5. Доказательство теоремы 1. Пусть $v \in J \delta(\gamma(r))$. Заметим вначале, что каждая из мер $\lambda_{+}(v)$ и $\lambda_{-}(v)$ имеет конечную $\gamma$-плотность. Мера $\lambda_{-}(v)$ имеет конечную $\gamma$-плотность по определению класса $J \delta(\gamma(r))$. То, что мера $\lambda_{+}(v)$ имеет конечную $\gamma$-плотность, следует из формулы (13). Из этой же формулы мы получаем, что

$$
\int_{0}^{\pi}\left|v\left(r e^{i \varphi}\right)\right| \sin \varphi d \varphi \leqslant A \gamma(B r) .
$$

Заметим также, что мера $|\lambda|=\lambda_{+}+\lambda_{-}$имеет конечную $\gamma$-плотность, а значит, удовлетворяет неравенству (15). Из (16) следует неравенство

$$
\left|c_{k}(r, v)\right| \leqslant k A \gamma(B r)
$$

Из формулы (1) получаем

$$
c_{k}(r, v)=\frac{1}{2^{k}} c_{k}(2 r, v)-\frac{2 r^{k}}{\pi} \int_{r}^{2 r} \frac{\lambda_{k}(t)}{t^{2 k+1}} d t
$$

откуда с учетом (3), (15), (17) следует неравенство

$$
\left|c_{k}(r, v)\right| \leqslant \frac{k}{2^{k}} A \gamma(2 B r)+\frac{2}{\pi} A \gamma(B r) .
$$

Тем самым, импликация 1) $\Longrightarrow 2$ ) доказана.

Пусть теперь выполнено условие 2) теоремы. Из неравенства $\left|c_{1}(r, v)\right| \leqslant A \gamma(B r)$ и формулы (2) следует, что если одна из мер $\lambda_{+}(v)$ или $\lambda_{-}(v)$ имеет конечную 
$\gamma$-плотность, то и другая мера имеет конечную $\gamma$-плотность, и, таким образом, мера $|\lambda|$ имеет конечную $\gamma$-плотность. Далее мы оценим $v_{+}(z)$, используя формулу $(4)$ при $R_{1}=r / 2, R_{2}=2 r$. Используя разложение в ряд Фурье (9), (10) при $q=1 / 2$, $R=r=|z|$, получим:

$$
\begin{aligned}
& \left|\frac{r}{\pi} \int_{0}^{\pi} \frac{\partial G\left(z, 2 r e^{i \varphi}\right)}{\partial n} v\left(2 r e^{i \varphi}\right) d \varphi+\frac{r}{4 \pi} \int_{0}^{\pi} \frac{\partial G\left(z,(r / 2) e^{i \varphi}\right)}{\partial n} v\left(\frac{1}{2} r e^{i \varphi}\right) d \varphi\right| \\
& \quad \leqslant \sum_{m=1}^{\infty} \frac{1}{2^{m}} \frac{4^{m}}{1+4^{m}}\left[2\left|c_{m}(2 r, v)\right|+8\left|c_{m}\left(\frac{1}{2} r, v\right)\right|\right] \leqslant A \gamma(B r)
\end{aligned}
$$

Из этого неравенства и формулы (4) получаем

$$
v_{+}(z) \leqslant \frac{1}{2 \pi} \iint_{D_{+}(r / 2,2 r)} K(z, \zeta) d \lambda_{-}(\zeta)+A \gamma(B r)
$$

Теперь, используя ортогональность системы полиномов $\{\sin k \theta\}, k=1,2, \ldots$, на отрезке $[0, \pi]$ и формулы (5)-(8), имеем

$$
\begin{aligned}
\int_{0}^{\pi} v_{+} & \left(r e^{i \theta}\right) \sin \theta d \theta \leqslant \frac{1}{2 \pi} \int_{0}^{\pi}\left\{\left[\iint_{D_{+}(r / 2, r)}+\iint_{D_{+}(r, 2 r)}\right] K(z, \zeta) d \lambda_{-}(\zeta)\right\} \\
& \times \sin \theta d \theta+2 A \gamma(B r) \leqslant \frac{1}{2} \iint_{D_{+}(r / 2, r)} \frac{\sin \varphi}{\operatorname{Im} \zeta} \frac{\tau}{r} \frac{4}{5}\left(1-\frac{r^{2}}{4 \tau^{2}}\right) d \lambda_{-}(\zeta) \\
& +\frac{1}{2} \iint_{D_{+}(r, 2 r)} \frac{\sin \varphi}{\operatorname{Im} \zeta} \frac{r}{\tau} \frac{4}{5}\left(1-\frac{\tau^{2}}{4 r^{2}}\right) d \lambda_{-}(\zeta)+2 A \gamma(B r) \\
\leqslant & \frac{2}{5 r} \int_{r / 2}^{r}\left(1-\frac{r^{2}}{4 \tau^{2}}\right) d \lambda_{-}(\tau)+\frac{2}{5 r} \int_{r}^{2 r}\left(1-\frac{\tau^{2}}{4 r^{2}}\right) d \lambda_{-}(\tau)+2 A \gamma(B r) \\
\leqslant & \frac{2}{5 r} \int_{r / 2}^{2 r} d \lambda_{-}(\tau)+2 A \gamma(B r) \leqslant \frac{2 \lambda_{-}(2 r)}{5 r}+2 A \gamma(B r)
\end{aligned}
$$

Отсюда следует, что функция $v \in J \delta(\gamma(r))$.

ТЕОРема 2. Пусть $\gamma-$ функция роста, $v \in J S$. Следующие утвержсдения әквивалентния:

1) $v \in J S(\gamma(r))$;

2) $\left|c_{k}(r, v)\right| \leqslant A \gamma(B r), k \in \mathbb{N}$, при некоторьх положительньх $A, B$ и всех $r>0$.

Эта теорема - непосредственное следствие теоремы 1, так как для функции класса $J S$ мера $\lambda_{-}$равна нулю.

В дополнение к теореме 1 покажем, что из условия 1) не следует такое усиление 3$):\left|c_{k}(r)\right| \leqslant \varepsilon_{k} \gamma(B r)$, где $\varepsilon_{k} \rightarrow 0$ при $k \rightarrow \infty$. Это показьвает следующий 
ПримеР. Рассмотрим неположительную, гармоническую в $\mathbb{C}_{+}$функцию

$$
v(z)=\sum_{k=-\infty}^{k=\infty} \operatorname{Im} \frac{2^{(\rho+1) k}}{z-2^{k}}, \quad 0<\rho<1 .
$$

Функция $v$ удовлетворяет соотношению $v(2 z)=2^{\rho} v(z)$. Действительно,

$$
v(2 z)=\sum_{k=-\infty}^{k=\infty} \operatorname{Im} \frac{2^{(\rho+1) k}}{2 z-2^{k}}=\frac{1}{2} \sum_{k=-\infty}^{k=\infty} \operatorname{Im} \frac{2^{(\rho+1) k}}{z-2^{k-1}}=2^{\rho} \sum_{k=-\infty}^{k=\infty} \operatorname{Im} \frac{2^{(\rho+1) k}}{z-2^{k}} .
$$

Отсюда получаем

$$
C_{1} r^{\rho} \leqslant \int_{0}^{\pi}\left|v\left(r e^{i \varphi}\right)\right| \sin \varphi d \varphi \leqslant C_{2} r^{\rho}
$$

где

$$
\begin{aligned}
& C_{1}=2^{-\rho} \inf _{1 \leqslant r \leqslant 2}\left(-\int_{0}^{\pi} v\left(r e^{i \theta}\right) \sin \theta d \theta\right), \\
& C_{2}=2^{\rho} \sup _{1 \leqslant r \leqslant 2}\left(-\int_{0}^{\pi} v\left(r e^{i \theta}\right) \sin \theta d \theta\right) .
\end{aligned}
$$

Пусть $z=r e^{i \theta}, 2^{k_{0}}<r<2^{k_{0}+1}$. Из разложения

$$
v\left(r e^{i \theta}\right)=-\sum_{2^{k}<r} 2^{\rho(k+1)} \sum_{m=0}^{\infty} \frac{2^{m k} \sin (m+1) \theta}{r^{m+1}}-\sum_{2^{k}>r} 2^{\rho k} \sum_{m=1}^{\infty} \frac{r^{m} \sin m \theta}{2^{m k}}
$$

непосредственньм подсчетом убеждаемся в справедливости соотношений

$$
\begin{aligned}
c_{n}(r, v) & =-\frac{1}{r^{n}} \sum_{2^{k}<r} 2^{k(\rho+n)}-r^{n} \sum_{2^{k}>r} 2^{k(\rho-n)} \\
& =-\frac{1}{r^{n}} \sum_{k=-\infty}^{k_{0}} 2^{k(\rho+n)}-r^{n} \sum_{k=k_{0}+1}^{\infty} 2^{k(\rho-n)} \\
& =-\frac{1}{r^{n}} \frac{2^{k_{0}(\rho+n)}}{1-2^{-(\rho+n)}}-r^{n} \frac{2^{\left(k_{0}+1\right)(\rho-n)}}{1-2^{\rho-n}} .
\end{aligned}
$$

В силу утверждения 3.1 эти соотношения имеют место и при $r=r_{0}=2^{k_{0}}$ :

$$
\begin{aligned}
c_{n}\left(r_{0}, v\right) & =-\frac{1}{2^{k_{0}}} \frac{2^{k_{0}(\rho+n)}}{1-2^{-(\rho+n)}}-2^{k_{0}} \frac{2^{\left(k_{0}+1\right)(\rho-n)}}{1-2^{\rho-n}} \\
& =-\frac{r_{0}^{\rho}}{1-2^{-(\rho+n)}}-\frac{2^{\rho} r_{0}^{\rho}}{2^{n}\left(1-2^{\rho-n}\right)} .
\end{aligned}
$$

Отсюда получаем неравенство

$$
\left|c_{n}\left(r_{0}, v\right)\right| \geqslant r_{0}^{\rho}, \quad n \in \mathbb{N} .
$$

Так как число $r_{0}=2^{k_{0}}$ может быть выбрано как угодно большим, то последнее неравенство показьвает невозможность усиления теоремы 1. 
6. Анализ мер в полуплоскости. Для описания полных мер суб- и $\delta$-субгармонических функций из классов $J \delta(\gamma(r))$ нам понадобятся некоторые специальные свойства мер в полуплоскости. В то время как понятие конечной $\gamma$-плотности характеризует меру круга, вводимое ниже понятие $\gamma$-сбалансированности будет характеризовать в некотором смысле распределение меры по аргументам. Совокупность же этих понятий и составит понятие $\gamma$-допустимости. Аналогичное понятие для мероморфных функций во всей плоскости рассматривали Л. Рубел и Б. Тейлор [1]. Как будет показано ниже, $\gamma$-допустимые меры, и только они, являются полными мерами функций из класса $S K(\gamma(r))$. Но доказательство этого факта нетривиально и требует еше одного понятия. Дело в том, что, как уже отмечалось, субгармоническая функция не определяется единственным образом своей полной мерой. Совокупность же $\lambda(v),\left\{\alpha_{k}(v)\right\}$ характеризует субгармоническую функцию $v$ единственньм образом. Поэтому нам придется изучать не одни лишш меры $\lambda$, а некие тандемы - пары $(\lambda, \alpha)$, определенным образом взвешенных относительно функции роста $\gamma$. В связи с этим по аналогии с коэффициентами Фурье субгармонической функции будут рассмотрены коэффициенты Фурье пары $(\lambda, \alpha)$.

Пусть $\lambda$-мера, удовлетворяющая условиям $\left\{\mathbf{G}^{+}\right\}, \gamma-$ некоторая функция роста. Для $k \in \mathbb{N}$ обозначим

$$
S_{+}(r ; k, \lambda)=\frac{1}{\pi k} \iint_{D_{+}\left(r_{0}, r\right)} \frac{\sin k \varphi}{\tau^{k} \operatorname{Im} \zeta} d \lambda(\zeta)+\frac{1}{\pi k r_{0}^{2 k}} \iint_{C_{+}\left(0, r_{0}\right)} \frac{\sin k \varphi}{\operatorname{Im} \zeta} \tau^{k} d \lambda(\zeta)
$$

где $\zeta=\tau e^{i \varphi}, r_{0}>0-$ фиксированное число,

$$
S_{+}\left(r_{1}, r_{2} ; k, \lambda\right)=S_{+}\left(r_{2} ; k, \lambda\right)-S_{+}\left(r_{1} ; k, \lambda\right), \quad r_{1} \leqslant r_{2}
$$

Будем иногда пользоваться сокрашенными обозначениями $S_{+}(r ; k)=S_{+}(r ; k, \lambda)$ и т.д.

Мера $\lambda$ называется $\gamma$-сбалансированной, если существуют положительные постоянные $A, B$, при которых

$$
S_{+}\left(r_{1}, r_{2} ; k, \lambda\right) \leqslant \frac{A \gamma\left(B r_{1}\right)}{r_{1}^{k}}+\frac{A \gamma\left(B r_{2}\right)}{r_{2}^{k}}
$$

для всех $r_{2}>r_{1}>0$ и $k \in \mathbb{N}, k \geqslant 2$.

Мера $\lambda$ называется $\gamma$-допустимой, если она $\gamma$-сбалансирована и имеет конечную $\gamma$-плотность. Условия $\gamma$-сбалансированности и конечной $\gamma$-плотности независимы. Заметим, что условия конечной $\gamma$-плотности и (18) при $k=1$ эквивалентны.

Мера $\lambda$ называется $\gamma$-взвешенной, если существуют последовательность вешественных чисел $\alpha=\left\{\alpha_{k}\right\}$ и положительные постоянные $A, B$, при которых для всех $r>0, k \in \mathbb{N}$ выполняется

$$
\left|\alpha_{k}+S_{+}(r ; k, \lambda)\right| \leqslant \frac{A \gamma(B r)}{r^{k}}
$$

Пусть $\gamma$ - функция роста. Положим $p[\gamma]=\infty$, если для всех $p \in \mathbb{N}$ выполняется

$$
\underset{r \rightarrow \infty}{\lim } \gamma(r) r^{-p}>0
$$

и

$$
p[\gamma]=\min \left\{p: p \in \mathbb{N}, \underset{r \rightarrow \infty}{\lim } \gamma(r) r^{-p}=0\right\}
$$

в противном случае. 
ТЕОРема 3. Пусть мера $\lambda$ имеет конечную $\gamma$-плотность. Тогда следующие утверэсдения эквивалентны:

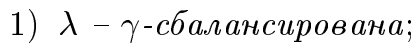

2) $\lambda-\gamma$-взвешена;

3) $\lambda-\gamma$-допустима.

ДокАЗАТЕЛЬСтво. Утверждения 1) и 3) эквивалентны по определению. Докажем, что из 1) следует 2). Для $1 \leqslant k<p[\gamma], k \in \mathbb{N}$, имеем

$$
\inf \left\{r^{-k} \gamma(B r): r>0\right\}>0
$$

поэтому при всех $k, 1 \leqslant k<p[\gamma] \leqslant \infty$, существует $r_{k}>0$ такое, что неравенство

$$
\frac{\gamma\left(B r_{k}\right)}{r_{k}^{k}} \leqslant 2 \frac{\gamma(B r)}{r^{k}}
$$

вьполняется для всех $r>0$. Для этих $k$ определим

$$
\alpha_{k}=-S_{+}\left(r_{k} ; k\right)
$$

Для $k \geqslant p[\gamma], p[\gamma]<\infty$, сушествует последовательность $\left\{r_{j}\right\}, r_{j} \uparrow \infty$ при $j \rightarrow \infty$, такая, что

$$
\lim _{j \rightarrow \infty} \gamma\left(B r_{j}\right) r_{j}^{-p[\gamma]}=0
$$

По предположению имеем

$$
S_{+}\left(r_{m}, r_{j} ; k,\right) \leqslant \frac{A \gamma\left(B r_{m}\right)}{r_{m}^{k}}+\frac{A \gamma\left(B r_{j}\right)}{r_{j}^{k}}
$$

Из (20) следует фундаментальность последовательности $\left\{S_{+}\left(r_{j} ; k\right)\right\}$. Для $k \geqslant p[\gamma]$ положим

$$
\alpha_{k}=-\lim _{j \rightarrow \infty} S_{+}\left(r_{j} ; k\right) .
$$

Таким образом, если $1 \leqslant k<p[\gamma]$, то

$$
\left|\alpha_{k}+S_{+}(r ; k)\right|=S_{+}\left(r_{k}, r ; k\right) \leqslant \frac{A \gamma\left(B r_{k}\right)}{r_{k}^{k}}+\frac{A \gamma(B r)}{r^{k}} \leqslant 3 \frac{A \gamma(B r)}{r^{k}} .
$$

Если же $k \geqslant p[\gamma]$, то

$$
\left|\alpha_{k}+S_{+}(r ; k)\right| \leqslant \lim _{j \rightarrow \infty} S_{+}\left(r, r_{j} ; k\right) \leqslant \frac{A \gamma(B r)}{r^{k}}+\lim _{j \rightarrow \infty} \frac{A \gamma\left(B r_{j}\right)}{r_{j}^{k}}=\frac{A \gamma(B r)}{r^{k}}
$$

Итак, мера $\lambda \gamma$-взвешена и импликация 1) $\Longrightarrow 2$ ) установлена. Из 2), в свою очередь, следует 1), так как

$\left|S_{+}\left(r_{1}, r_{2} ; k\right)\right|=\left|S_{+}\left(r_{2} ; k\right)+\alpha_{k}-\alpha_{k}-S_{+}\left(r_{1} ; k\right)\right| \leqslant\left|\alpha_{k}+S_{+}\left(r_{1} ; k\right)\right|+\left|\alpha_{k}+S_{+}\left(r_{2} ; k\right)\right|$.

Теорема 3, таким образом, полностью доказана.

Рассмотрим отдельно случай, когда $\gamma(r)=r^{\rho(r)}=V(r)$, где $\rho(r)$ - уточненньй порядок в смысле Валирона, $\lim _{r \rightarrow \infty} \rho(r)=\rho \leqslant 1$. 
ЛЕмма 3. Пусть $\gamma(r)=V(r), \lambda-м е р а$, удовлетворяющая условиям $\left\{\mathbf{G}^{+}\right\}$. Справедливы такие утверждения:

1) мера $\lambda$ имеет конечную $\gamma$-плотность тогда и только тогда, когда

$$
\varlimsup_{r \rightarrow \infty} \lambda(r)\{r V(r)\}^{-1}<\infty
$$

2) если $\rho$ не челое, то каждая мера $\lambda$ с конечной $\gamma$-плотностью является $\gamma$-допустимой;

3) если $\rho$ челое, то $\lambda$ ү-допустима тогда и только тогда, когда она имеет конечную $\gamma$-плотность, $u \frac{1}{L(r)}\left[\alpha_{\rho}+S_{+}(r ; \rho, \lambda)\right]$ является ограниченной функцией от $r$, әде $L(r)=V(r) / r^{\rho}, \alpha_{\rho}$ - постоянное число.

ДоказАТЕльство. 1) Если $\lambda$ имеет конечную $\gamma$-плотность, то из леммы 2 и свойств уточненного порядка следует, что $\lambda(r)=O(V(r) r), r \rightarrow \infty$, а если $\lambda(r) \leqslant$ $A V(r) r, A>0$, то (см. [10; $\S 12$, свойство 3$])$ при $\rho>1$

$$
N(r, \lambda) \leqslant A \int_{r_{0}}^{r} \frac{V(t)}{t^{2}} d t=\frac{A}{\rho-1} \frac{V(r)}{r}+o\left(\frac{V(r)}{r}\right) .
$$

Случай $\rho=1$ относится к п. 3).

2) Так как $k \neq \rho$ при $k \in \mathbb{N}$, то, используя неравенство $\lambda(r) \leqslant A V(r) r$, при некотором $A>0$ имеем (считая, что $\left.r_{1} \leqslant r_{2}\right)$, как и в п. 1$)$,

$$
\begin{gathered}
\left|S_{+}\left(r_{1}, r_{2} ; k, \lambda\right)\right| \leqslant \frac{1}{\pi} \int_{r_{1}}^{r_{2}} \frac{d \lambda(t)}{t^{k+1}}=\frac{1}{\pi}\left[\frac{\lambda\left(r_{2}\right)}{r_{2}^{k+1}}-\frac{\lambda\left(r_{1}\right)}{r_{1}^{k+1}}+(k+1) \int_{r_{1}}^{r_{2}} \frac{\lambda(t)}{t^{k+2}} d t\right] \\
\leqslant \frac{A V\left(r_{2}\right)}{r_{2}^{k}}+A(k+1) \int_{r_{1}}^{r_{2}} \frac{V(t)}{t^{k+1}} d t \leqslant A \frac{k+1}{|\rho-k|}\left(\frac{V\left(r_{2}\right)}{r_{2}^{k}}+\frac{V\left(r_{1}\right)}{r_{1}^{k}}\right),
\end{gathered}
$$

т.е. $\lambda$-сбалансирована. По теореме 3 она является $\gamma$-допустимой.

3) Пусть $\rho$ целое, мере $\lambda$ присуща конечная $\gamma$-плотность и $\frac{1}{L(r)} S_{+}(r ; \rho, \lambda)$ - ограниченная функция от $r$. При $k \neq \rho$ имеем $(22)$. Но так как функция $\frac{1}{L(r)}\left[\alpha_{\rho}+\right.$ $\left.S_{+}(r ; \rho, \lambda)\right]$ ограничена некоторым числом $B>0$, то

$$
\begin{aligned}
\left|S_{+}\left(r_{1}, r_{2} ; \rho, \lambda\right)\right| & \leqslant\left|\alpha_{\rho}+S_{+}\left(r_{1} ; \rho, \lambda\right)\right|+\left|\alpha_{\rho}+S_{+}\left(r_{2} ; \rho, \lambda\right)\right| \\
& \leqslant B\left(L\left(r_{1}\right)+L\left(r_{2}\right)\right)=B\left(\frac{V\left(r_{2}\right)}{r_{2}^{\rho}}+\frac{V\left(r_{1}\right)}{r_{1}^{\rho}}\right) .
\end{aligned}
$$

Отсюда и из (22) получаем, что $\lambda \gamma$-сбалансирована. На основании теоремы 3 она является $\gamma$-допустимой. Обратное следует из теоремы 3.

Для $k \in \mathbb{N}$ и меры $\lambda$, удовлетворяющей условиям $\left\{\mathbf{G}^{+}\right\}$, обозначим

$$
S_{+}^{\prime}(r ; k)=S_{+}^{\prime}(r ; k, \lambda)=\frac{1}{\pi k r^{k}} \iint_{C_{+}(0, r)} \frac{\tau^{k} \sin k \varphi}{\operatorname{Im} \zeta} d \lambda(\zeta), \quad \zeta=\tau e^{i \varphi} .
$$


Лемма 4. Справедливо неравенство

$$
\left|S_{+}^{\prime}(r, k)\right| \leqslant \frac{N(e r, \lambda) r e^{2}}{\pi} .
$$

ДокАЗАтЕльство. Так же, как и при доказательстве леммы 2, имеем

$$
\left|S_{+}^{\prime}(r, k)\right| \leqslant \frac{\lambda(r)}{\pi r} \leqslant \frac{N(e r, \lambda) r e^{2}}{\pi} .
$$

Пусть $\alpha=\left\{\alpha_{k}\right\}$ - некоторая последовательность вещественных чисел. Функции

$$
c_{k}(r ; \lambda, \alpha)=r^{k}\left\{\alpha_{k}+S_{+}(r ; k)\right\}-S_{+}^{\prime}(r ; k), \quad k \in \mathbb{N}
$$

называются коэффичиентами Фурье пары $(\lambda, \alpha)$.

Пара $(\lambda, \alpha)$ называется $\gamma$-допустимой, если существуют положительные постоянные $A, B$, при которых

$$
\left|c_{k}(r ; \lambda, \alpha)\right| \leqslant A \gamma(B r), \quad r>0, \quad k \in \mathbb{N},
$$

и мера $\lambda$ имеет конечную $\gamma$-плотность.

ТЕОРЕМА 4. Мера $\lambda$ является $\gamma$-допустимой тогда и только тогда, когда существует такая последовательность $\alpha$, что пара $(\lambda, \alpha) \gamma$-допустима.

ДоказАтЕльство. Пусть $\lambda \gamma$-допустима. Тогда по теореме 3 она $\gamma$-взвешена, и мы можем взять в качестве $\alpha$ последовательность, входящую в определение $\gamma$-взвешенности. Тогда соотношение (25) следует при $k \in \mathbb{N}$ из неравенства

$$
\left|c_{k}(r ; \lambda, \alpha)\right| \leqslant r^{k}\left|\alpha_{k}+S_{+}(r ; k)\right|+\left|S_{+}^{\prime}(r ; k)\right|
$$

леммы 4 и выбора последовательности $\alpha$.

Предположим теперь, что вьполнено соотношение (25). Тогда при $k \in \mathbb{N}$ имеем

$$
r^{k}\left|\alpha_{k}+S_{+}(r ; k)\right|=\left|c_{k}(r ; \lambda, \alpha)+S_{+}^{\prime}(r ; k)\right| \leqslant A \gamma(B r)+\frac{N(r, \lambda) r}{\pi \ln 2} \leqslant A_{1} \gamma(B r)
$$

Таким образом, мера $\lambda$ является $\gamma$-взвешенной. По теореме 3 она $\gamma$-допустима.

7. Описание полных мер суб- и $\delta$-субгармонических функций конечного $\gamma$-типа. Целью настоящего пункта является, во-первых, установление двух критериев, характеризуюших полные меры суб- и $\delta$-субгармонических функций конечного $\gamma$-типа. Для этого доказана теорема о существовании субгармонической функции с заданной последовательностью $\left\{c_{k}(r ; \lambda, \alpha)\right\}=\left\{c_{k}(r ; v)\right\}$, а также использован анализ мер, развитый в предыдушем пункте.

Во-вторых, рассматривается проблема о представлении $\delta$-субгармонической функции $v \in J \delta(\gamma(r))$ в виде разности двух субгармонических функций из класса $J S(\gamma(r))$. Решение этой проблемы для мероморфных во всей плоскости функций принадлежит Д. Майлзу [2]. 
ТЕОРема 5. Пусть $c_{k}(r)=c_{k}(r ; \lambda, \alpha)-$ коэффичиенты Фурье парьь $(\lambda, \alpha)$, удовлетворяющие условию

$$
\sup _{k \in \mathbb{N}}\left|c_{k}(r)\right| \leqslant C(r), \quad r>0
$$

Тогда существует единственная субгармоническая функиия $v$, для которой полная мера $\lambda(v)=\lambda u c_{k}(r ; v)=c_{k}(r)$ для всех $k \in \mathbb{N} u$ всех $r>0$.

ДокАЗАТЕЛЬство. Прежде всего докажем вспомогательную лемму.

Лемма 5. Пусть $v_{1}, v_{2}$ - субгармонические функции в полукруге $C_{+}(0, R)$, имеющие при всех $k \in \mathbb{Z}$ одинаковые коэффициенты Фурье. Тогда $v_{1} \equiv v_{2}$.

ДокАЗАТЕЛьство. Так как при любом $r, 0<r<R$, коэффициенты Фурье функций $v_{1}$ и $v_{2}$ равны, то почти для всех $\theta \in[0, \pi]$ при любом фиксированном $r$ имеет место $v_{1}\left(r e^{i \theta}\right)=v_{2}\left(r e^{i \theta}\right)$ (см. [12; гл. 2, п. 2.4]). У тверждение леммы тогда следует из равенства (см., например, [11])

$$
v(z)=\lim _{\varepsilon \rightarrow 0} \frac{1}{\pi \varepsilon^{2}} \iint_{|\zeta-z| \leqslant \varepsilon} v(\zeta) d s(\zeta),
$$

где $d s(\zeta)$ - элемент площади. Это можно получить переходом к повторному интегралу

$$
v(z)=\lim _{\varepsilon \rightarrow 0} \frac{1}{\pi \varepsilon^{2}} \iint v\left(z+\rho e^{i \theta}\right) \rho d \rho d \theta
$$

по области $|z|-\varepsilon \leqslant \rho \leqslant|z|+\varepsilon, \theta_{1}(\rho) \leqslant \theta \leqslant \theta_{2}(\rho)$.

Докажем теперь теорему. Определим

$$
\Phi\left(\rho e^{i \varphi}\right)=\sum_{k=1}^{\infty} c_{k}(\rho) \sin k \varphi
$$

Согласно (26) для каждого $\rho>0$ функция $\Phi\left(\rho e^{i \varphi}\right) \in \mathbf{D}^{2}$ (см., например, [13]), где $\mathbf{D}^{2}$ - множество распределений порядка не выше 2. Для $\rho>0$ полагаем

$$
\begin{gathered}
P_{\rho}(z)=-\frac{1}{2 \pi} \iint_{\frac{C_{+}(0, \rho)}{}} K(z, \zeta) d \lambda(\zeta), \quad a_{\rho}(z)=\frac{\rho}{2 \pi} \int_{0}^{\pi} \frac{\partial G\left(z, \rho e^{i \varphi}\right)}{\partial n} \Phi\left(\rho e^{i \varphi}\right) d \varphi \\
v_{\rho}(z)=a_{\rho}(z)+P_{\rho}(z)
\end{gathered}
$$

где $G$ - функция Грина полукруга $C_{+}(0, \rho)$.

Функция $v_{\rho}(z)$, очевидно, субгармонична в полукруге $C_{+}(0, \rho)$. Покажем, что $c_{k}\left(r ; v_{\rho}\right)=c_{k}(r)$ при $r \leqslant \rho, k \in \mathbb{N}$. Обозначим

$$
P_{\rho}\left(r e^{i \theta}\right)=\sum_{k=1}^{\infty} d_{k}(r) \sin k \theta
$$


Пусть $k \in \mathbb{N}$. Тогда, используя формулу

$$
\frac{\partial G\left(r e^{i \theta}, \rho e^{i \varphi}\right)}{\partial n}=4 \sum_{m=1}^{\infty} \frac{r^{m}}{\rho^{m+1}} \sin m \theta \sin m \varphi
$$

и находя коэффициенты Фурье функции $a_{\rho}\left(r e^{i \theta}\right)$, получаем

$$
c_{k}\left(r, v_{\rho}\right)=\left(\frac{r}{\rho}\right)^{k} c_{k}(\rho)+d_{k}(r) .
$$

Коэффициенты Фурье функции $P_{\rho}\left(r e^{i \theta}\right)$ находятся из формулы (12):

$$
\begin{aligned}
d_{k}(r)= & \alpha_{k}\left(P_{\rho}\right)+\frac{r^{k}}{\pi k r_{0}^{2 k}} \iint_{\overline{C_{+}\left(0, r_{0}\right)}} \frac{\sin k \varphi}{\operatorname{Im} \zeta} \tau^{k} d \lambda(\zeta) \\
& +\frac{r^{k}}{\pi k} \iint_{D_{+}\left(r_{0}, r\right)} \frac{\sin k \varphi}{\operatorname{Im} \zeta \tau^{k}} d \lambda(\zeta)-\frac{1}{r^{k} \pi k} \iint_{\frac{C_{+}(0, r)}{\operatorname{Im} \zeta}} \frac{\sin k \varphi}{k} d \lambda(\zeta) .
\end{aligned}
$$

Из формулы (1), интегрируя по частям и учитывая, что $P_{\rho}\left(\rho e^{i \theta}\right)=0, \theta \in[0, \pi]$, находим

$$
\begin{aligned}
\alpha_{k}\left(P_{\rho}\right)=- & \frac{2}{\pi} \int_{r_{0}}^{\rho} \frac{\lambda_{k}(t)}{t^{2 k+1}} d t=-\frac{1}{\pi k r_{0}^{2 k}} \iint_{\overline{C_{+}\left(0, r_{0}\right)}} \frac{\sin k \varphi}{\operatorname{Im} \zeta} \tau^{k} d \lambda(\zeta) \\
& -\frac{1}{\pi k} \iint_{D_{+}\left(r_{0}, \rho\right)} \frac{\sin k \varphi}{\operatorname{Im} \zeta \tau^{k}} d \lambda(\zeta)+\frac{1}{\rho^{2 k} \pi k} \iint_{\frac{C_{+}(0, \rho)}{\operatorname{Im} \zeta}} \frac{\sin k \varphi}{\tau^{k}} d \lambda(\zeta) .
\end{aligned}
$$

Подставляя это представление в выражение для $d_{k}(r)$, получим

$$
\begin{aligned}
d_{k}(r)= & -\frac{1}{r^{k} \pi k} \iint_{C_{+}(0, r)} \frac{\sin k \varphi}{\operatorname{Im} \zeta} \tau^{k} d \lambda(\zeta) \\
& -\frac{r^{k}}{\pi k} \iint_{D_{+}(r, \rho)} \frac{\sin k \varphi}{\operatorname{Im} \zeta \tau^{k}} d \lambda(\zeta)+\frac{r^{k}}{\pi k \rho^{2 k}} \iint_{C_{+}(0, r)} \frac{\sin k \varphi}{\operatorname{Im} \zeta} \tau^{k} d \lambda(\zeta) .
\end{aligned}
$$

Кроме того, по определению коэффициентов Фурье пары имеем

$$
\begin{aligned}
\left(\frac{r}{\rho}\right)^{k} c_{k}(\rho)= & r^{k}\left[\alpha_{k}+\frac{1}{\pi k r_{0}^{2 k}} \iint_{\frac{C_{+}\left(0, r_{0}\right)}{\operatorname{Im} \zeta}} \frac{\sin k \varphi}{k} d \lambda(\zeta)\right. \\
& \left.+\frac{1}{\pi k} \iint_{D_{+}\left(r_{0}, \rho\right)} \frac{\sin k \varphi}{\operatorname{Im} \zeta \tau^{k}} d \lambda(\zeta)\right]-\frac{r^{k}}{\pi k \rho^{2 k}} \iint_{\frac{C_{+}(0, \rho)}{\operatorname{Im} \zeta}} \frac{\sin k \varphi}{\operatorname{Im}} d \lambda(\zeta)
\end{aligned}
$$

Подставляя это выражение и выражение для $d_{k}(r)$ в $(27)$, получаем требуемое равенство для $k \in \mathbb{N}$.

Если $\rho^{\prime}>\rho$, то функция $v_{\rho^{\prime}}-$ продолжение функции $v_{\rho}$. Действительно, при $0 \leqslant r<\rho c_{k}\left(r, v_{\rho}\right)=c_{k}(r)=c_{k}\left(r, v_{\rho^{\prime}}\right)$ и по лемме $5 v_{\rho}(z) \equiv v_{\rho^{\prime}}(z)$, если $z \in C_{+}(0, \rho)$.

Положим теперь $v(z)=v_{\rho}(z)$ при $|z|<\rho$. Очевидно, функция $v(z)$ субгармоническая и удовлетворяет требованиям теоремы. Единственность ее следует из леммы 5. Теорема доказана. 
ТЕОрема 6. Для того чтобъ мера $\lambda$ бъла полной мерой истинно субгармонической функции $v$ конечного $\gamma$-типа, необходимо и достаточно, чтобъ $\lambda$ бъла $\gamma$-допустимой.

ДокАЗАТЕЛьство. Если $\lambda=\lambda(v)$ для некоторой функции $v \in J S(\gamma(r))$, то по теореме 3 пара $(\lambda, \alpha(v))$, где $\alpha(v)=\left\{\alpha_{k}\right\}$ - последовательность из (12), является $\gamma$-допустимой. Согласно теореме 4 мера $\lambda \gamma$-допустима.

Предположим, что $\lambda-\gamma$-допустимая мера. На основании теоремы 4 сушествует последовательность $\alpha$, при которой пара $(\lambda, \alpha) \gamma$-допустима. По теореме 5 существует единственная субгармоническая функция $v$, для которой $\lambda(v)=\lambda, \alpha(v)=\alpha$ и $\left\{c_{k}(r, v)\right\}=\left\{c_{k}(r, \lambda, \alpha)\right\}, r>0$. В соответствии с теоремой $2 v \in J S(\gamma(r))$. Тем самьм, теорема 6 полностью доказана.

ТЕОРема 7. Пусть $\lambda$-мера, $\rho(r)$ - уточненный порядок, $\lim _{r \rightarrow \infty} \rho(r)=$ $\rho \geqslant 1$. Если $\rho$ не челое, то $\lambda$ является полной мерой некоторой субгармонической функции порядка роста не выше $\rho(r)$ и нормального типа тогда и только тогда, когда

(*) существует постоянная $A>0$ такая, что $\lambda(r) \leqslant A V(r) r$ для всех $r>0$.

При иелом $\rho$ это имеет место тогда и только тогда, когда выполняетcя $(*) u$

$$
\frac{1}{L(r)}\left|\alpha_{\rho}+\frac{1}{\pi \rho} \iint_{D_{+}\left(r_{0}, r\right)} \frac{\sin \rho \varphi}{\tau^{\rho} \operatorname{Im} \zeta} d \lambda(\zeta)\right| \leqslant B
$$

при некоторых постоянных $\alpha_{\rho}, r_{0}>0, B>0$ и всех $r>r_{0}$.

Эта теорема вытекает из теоремы 6 и леммы 3.

Введем некоторые понятия. Суммой двух мер $\lambda_{1}$ и $\lambda_{2}$ называется мера $\lambda=$ $\lambda_{1}+\lambda_{2}$ такая, что $\lambda(G)=\lambda_{1}(G)+\lambda_{2}(G)$ для каждого $\lambda_{i}$-измеримого множества $G$ $(i=1,2)$. Сложение мер коммутативно и ассоциативно. При этом, очевидно, $\lambda(r)=\lambda_{1}(r)+\lambda_{2}(r)$. Если носитель меры $\lambda^{\prime}$ принадлежит носителю меры $\lambda$ и при этом $\lambda^{\prime}(G) \leqslant \lambda(G)$, то будем писать $\lambda^{\prime} \leqslant \lambda$. Как следует из леммы 1 , разность двух функций из $J S(\gamma(r))$ принадлежит классу $J \delta(\gamma(r))$. Теперь мы устанавливаем обратное утверждение: каждая функция $v$ из класса $J \delta(\gamma(r))$ представляется в виде разности двух функций $v_{1}$ и $v_{2}$ из класса $J S(\gamma(r))$.

Класс элементов $v_{1}-v_{2}, v_{1}, v_{2} \in J S(\gamma(r))$, обозначим через $J S(\gamma(r))-J S(\gamma(r))$.

TеОРема 8. Kлассы $J \delta(\gamma(r))$ u $J S(\gamma(r))-J S(\gamma(r))$ совпадают.

ДокАЗАТЕльство. Как мы заметили вьше, $J S(\gamma(r))-J S(\gamma(r)) \subset J \delta(\gamma(r))$. Докажем обратное: $J \delta(\gamma(r)) \subset J S(\gamma(r))-J S(\gamma(r))$.

Пусть $v \in J \delta(\gamma(r)), \lambda(v)=\lambda_{+}-\lambda_{-}$. Достаточно доказать сушествование $\gamma$-допустимой меры $\tilde{\lambda} \geqslant \lambda_{-}$. Действительно, если таковая существует, то согласно теореме 6 сушествует субгармоническая функция $v_{1} \in J S(\gamma(r))$, для которой $\lambda\left(v_{1}\right)=\tilde{\lambda}$. Тогда функция $v_{2}=v+v_{1}$ субгармоническая и $v_{2} \in J S(\gamma(r))$ на основании леммы 1 . Но $v=v_{2}-v_{1}$, и мы получаем требуемое представление.

Перейдем к построению такой меры $\tilde{\lambda}$. Обозначим

$$
B_{n}=\left\{z: 2^{n}<|z| \leqslant 2^{n+1}\right\} \cap \overline{\mathbb{C}}_{+}, \quad q=\sum_{m=1}^{\infty} \frac{m^{2}}{2^{m}} .
$$


Положим

$$
\begin{gathered}
g_{n}(\theta)=-\sum_{m=1}^{\infty} \frac{\sin m \theta}{2^{m} \sin \theta} \iint_{B_{n}} \frac{2^{m n} \sin m \varphi}{\tau^{m} \operatorname{Im} \zeta} d \lambda_{-}(\zeta), \quad \zeta=\tau e^{i \varphi}, \\
p_{n}=\iint_{B_{n}} \frac{d \lambda_{-}(\zeta)}{\tau}=\int_{2^{n}}^{2^{n+1}} \frac{d \lambda_{-}(t)}{t}, \quad f_{n}(\theta)=\sin \theta\left[g_{n}(\theta)+q p_{n}\right] .
\end{gathered}
$$

Поскольку

$$
\left|g_{n}(\theta)\right| \leqslant \sum_{m=1}^{\infty} \frac{m^{2}}{2^{m}} \iint_{B_{n}} \frac{d \lambda_{-}(\zeta)}{\tau}=q p_{n}
$$

TO

$$
0 \leqslant f_{n}(\theta) \leqslant 2 q \int_{2^{n}}^{2^{n+1}} \frac{d \lambda_{-}(t)}{t}
$$

При $k \geqslant 2, k \in \mathbb{N}$, имеем

$$
-\int_{0}^{\pi} \sin k \theta f_{n}(\theta) d \theta=\frac{\pi}{2} 2^{k(n-1)} \iint_{B_{n}} \frac{\sin k \varphi}{\tau^{k} \operatorname{Im} \zeta} d \lambda_{-}(\zeta)
$$

т.е.

$$
\iint_{B_{n}} \frac{\sin k \varphi}{\tau^{k} \operatorname{Im} \zeta} d \lambda_{-}(\zeta)+\frac{2^{-k(n-1)} 2}{\pi} \int_{0}^{\pi} \sin k \theta f_{n}(\theta) d \theta=0
$$

Положим

$$
L_{n}=\left[\frac{1}{\pi^{2}} \int_{0}^{\pi} f_{n}(\theta) d \theta\right]
$$

где [ $\cdot]$ означает целую часть. Поскольку $f_{n}(\theta) \geqslant 0$, то существуют возрастающие точки $\theta_{j}^{\prime}$ такие, что

$$
\frac{1}{\pi^{2}} \int_{0}^{\theta_{j}^{\prime}} f_{n}(\theta) d \theta=j, \quad j=1, \ldots, L_{n}
$$

причем $\theta_{j}^{\prime}$ зависят от $n$. Пусть

$$
W_{n}=\left\{b_{j}: b_{j}=2^{n-1} e^{i \theta_{j}^{\prime}}, j=1, \ldots, L_{n}\right\}, \quad W=\bigcup_{n \geqslant 1} W_{n},
$$

$\lambda^{\prime}$ - мера, сосредоточенная в точках множества $W, d \lambda^{\prime}\left(b_{j}\right)=2 \pi \operatorname{Im} b_{j}$. Тогда мера $\widetilde{\lambda}=\lambda_{-}+\lambda^{\prime}$ искомая. Установим это. Из (28) следует, что

$$
L_{n} \leqslant \frac{2 q}{\pi} \int_{2^{n}}^{2^{n+1}} \frac{d \lambda_{-}(t)}{t}
$$


При $2^{n-1}<r \leqslant 2^{n}$ имеем

$$
\begin{aligned}
\int_{r}^{2 r} \frac{d \lambda^{\prime}(t)}{t^{2}} & \leqslant \frac{2 \pi}{r} L_{n+1} \leqslant \frac{4 q}{r} \int_{2^{n+1}}^{2^{n+2}} \frac{d \lambda_{-}(t)}{t} \leqslant 32 q \int_{2^{n+1}}^{2^{n+2}} \frac{d \lambda_{-}(t)}{t^{2}} \\
& \leqslant 32 q\left(\int_{r}^{2 r}+\int_{2 r}^{4 r}\right) \frac{d \lambda_{-}(t)}{t^{2}}
\end{aligned}
$$

а поскольку у меры $\lambda_{-} \gamma$-плотность конечная, то и мера $\lambda^{\prime}$ имеет конечную $\gamma$-плотность, так как при любых $r_{0}<r$

$$
\int_{r_{0}}^{r} \frac{d \lambda^{\prime}(t)}{t^{2}} \leqslant 64 q \int_{r_{0}}^{2 r} \frac{d \lambda_{-}(t)}{t^{2}}
$$

Согласно (29)

$$
\begin{aligned}
& \frac{1}{\pi k}\left|\iint_{B_{n}} \frac{\sin k \varphi}{\tau^{k} \operatorname{Im} \zeta} d \lambda_{-}(\zeta)+\iint_{B_{n}} \frac{\sin k \varphi}{\tau^{k} \operatorname{Im} \zeta} d \lambda_{-}^{\prime}(\zeta)\right| \\
& \quad=\frac{1}{\pi k}\left|2 \pi \sum_{j=1}^{L_{n}} \frac{\sin k \theta_{j}^{\prime}}{2^{k(n-1)}}-\frac{2}{\pi 2^{k(n-1)}} \int_{0}^{\pi} \sin k \theta f_{n}(\theta) d \theta\right| .
\end{aligned}
$$

Обозначим это выражение через $\delta_{k n}$, разобъем интеграл, входящий в него, на сумму интегралов по промежуткам $\left[\theta_{j-1}^{\prime}, \theta_{j}^{\prime}\right], j=1, \ldots, L_{n}, \theta_{0}^{\prime}=0$, и промежутку $\left[\theta_{L_{n}}^{\prime}, \pi\right]$, а затем к каждому из них применим теорему о среднем значении. Учитывая равенства

$$
\frac{1}{\pi^{2}} \int_{\theta_{j-1}^{\prime}}^{\theta_{j}^{\prime}} f_{n}(\theta) d \theta=1, \quad j=1, \ldots, L_{n}
$$

получаем

$$
\begin{aligned}
\delta_{k n} & =\frac{2}{k 2^{k(n-1)}}\left|\sum_{j=1}^{L_{n}} \sin k \theta_{j}^{\prime}-\sum_{j=1}^{L_{n}} \frac{1}{\pi^{2}} \int_{\theta_{j-1}^{\prime}}^{\theta_{j}^{\prime}} \sin k \theta f_{n}(\theta) d t+\frac{1}{\pi^{2}} \int_{\theta_{L_{n}}^{\prime}}^{\pi} \sin k \theta f_{n}(\theta) d t\right| \\
& =\frac{2}{k 2^{k(n-1)}}\left|\sum_{j=1}^{L_{n}}\left(\sin k \theta_{j}^{\prime}-\sin k \varphi_{j}\right)+\frac{1}{\pi^{2}} \int_{\theta_{L_{n}}^{\prime}}^{\pi} \sin k \theta f_{n}(\theta) d t\right| \\
& \leqslant \frac{2}{k 2^{k(n-1)}}\left[\sum_{j=1}^{L_{n}}\left|\sin k \theta_{j}^{\prime}-\sin k \varphi_{j}\right|+1\right]
\end{aligned}
$$

где $\theta_{j}^{\prime} \leqslant \varphi_{j} \leqslant \theta_{j}, j=1, \ldots, L_{n}$.

Поскольку

$$
\left|\sin k \theta_{j}^{\prime}-\sin k \varphi_{j}\right| \leqslant k\left(\theta_{j}^{\prime}-\theta_{j-1}^{\prime}\right), \quad j=1, \ldots, L_{n}
$$

TO

$$
\delta_{k n} \leqslant \frac{2 \pi+1}{2^{k(n-1)}}
$$


Таким образом, если $2^{n-1} \leqslant r_{1}<r_{2} \leqslant 2^{n+1}$, то, поскольку мера $\widetilde{\lambda}$ имеет конечную $\gamma$-плотность, получим

$$
\left|S_{+}\left(r_{1}, r_{2} ; k, \tilde{\lambda}\right)\right|=\frac{1}{k \pi}\left|\iint_{r_{1} \leqslant \tau \leqslant r_{2}} \frac{\sin k \varphi}{\tau^{k} \operatorname{Im} \zeta} d \tilde{\lambda}(\zeta)\right| \leqslant \frac{1}{\pi r_{1}^{k}} \int_{r_{1}}^{4 r_{1}} \frac{d \widetilde{\lambda}(t)}{t} \leqslant \frac{A \gamma\left(B r_{1}\right)}{r_{1}^{k}}
$$

где $A$ и $B$ - некоторые постоянные, не зависящие от $n$ и $k$.

Если же $2^{m} \leqslant r_{1} \leqslant 2^{m+1}, 2^{n} \leqslant r_{2} \leqslant 2^{n+1}, m<n-1$, то, учитьвая определение $\delta_{k n}$ и оценку для него, находим

$$
\left|S_{+}\left(r_{1}, r_{2} ; k, \widetilde{\lambda}\right)\right| \leqslant \sum_{l=m+2}^{n} \delta_{k l}+\int_{r_{1}}^{4 r_{1}} \frac{d \widetilde{\lambda}(t)}{t^{k+1}}+\int_{r_{2}}^{2 r_{2}} \frac{d \widetilde{\lambda}(t)}{t^{k+1}}
$$

Так как

$$
\sum_{l=m+2}^{n} \delta_{k l} \leqslant(2 \pi+1) \sum_{l=m+2}^{n} \frac{1}{2^{k(l-1)}} \leqslant \frac{2(2 \pi+1)}{2^{k(m+1)}} \leqslant \frac{2(2 \pi+1)}{r_{1}^{k}}
$$

то, учитывая, что $\widetilde{\lambda}$ имеет конечную $\gamma$-плотность, получаем отсюда $\gamma$-сбалансированность меры $\widetilde{\lambda}$. А значит, $\widetilde{\lambda} \gamma$-допустима.

Теорема доказана.

Заключение. На протяжении статьи мы предполагали при определении классов растущих функций, что функция роста $\gamma(r)$ удовлетворяет условию (14). Оно необходимо, если мы хотим использовать для определения роста классическую неванлинновскую характеристику $T(r, v)$, так как неравенство $T(r, v) \leqslant A \gamma(B r) / r$ уже накладывает это ограничение на функцию роста $\gamma$. Если мы хотим рассматривать общий случай, без всяких ограничений на функцию роста (например, случай $\gamma(r)=r^{\rho}, 0<\rho<1$ ), то мы должны использовать более сложную характеристику, чем неванлинновская, а именно $v \in J \delta(\gamma(r))$, если

$$
m\left(r_{2}, v\right)+m\left(r_{1},-v\right)+\int_{r_{1}}^{r_{2}} \frac{\lambda_{-}(t)}{t^{3}} d t \leqslant \frac{A}{r_{1}} \gamma\left(B r_{1}\right)+\frac{A}{r_{2}} \gamma\left(B r_{2}\right)
$$

для всех $r_{2}>r_{1}>0$. В этом случае все утверждения нашей работы имеют место, а их доказательство автоматически повторяет приведенные вьше рассуждения.

Заметим, что это определение и определение, данное выше, совпадают, если функция роста удовлетворяет условию (14).

Введенное в статье определение порядка в случае, когда функция $v$ является субгармонической в полуплоскости и $\gamma(r)=r^{\rho}$, совпадает с определением, введенньгм Л. И. Ронкиньм [14], и отличается от определения формального порядка, введенного А.Ф. Гришиным [6], и эквивалентных между собой определений порядка Е. Титчмарша и Н. В. Говорова [15]. Однако все эти понятия совпадают при $\rho>1$. Качественное их отличие возникает при $\rho \leqslant 1$. В этом случае наше определение является наиболее широким, т.е. субгармоническая функция конечного порядка в смысле А. Ф. Гришина или Титчмарша-Говорова является функцией конечного порядка в смысле определения, данного в статье. 


\section{Список литературы}

1. Rubel L. A., Taylor B. A. Fourier series method for meromorphic and entire functions // Bull. Soc. Math. France. 1968. V. 96. P. 53-96.

2. Miles J. B. Quotient representations of meromorphic functions // J. Anal. Math. 1972. V. 25. P. 371-388.

3. Кондратюк $A$. A. Метод рядов Фурье для целых и мероморфных функций вполне регулярного роста. I // Матем. сб. 1978. Т. 106(148). №3. С. 386-408.

4. Кондратюк A. А. Метод рядов Фурье для целых и мероморфных функций вполне регулярного роста. II // Матем. сб. 1980. Т. 113(155). №1. С. 118-132.

5. Кондратюк $A$. А. Метод рядов Фурье для целшх и мероморфных функций вполне регулярного роста. III // Матем. сб. 1983. Т. 120(162). № 3. С. 331-343.

6. Гришин A. $\Phi$. Непрерьвность и асимптотическая непрерывность субгармонических функций // Матем. физика, анализ, геометрия. 1994. Т. 1. № 2. С. 193-215.

7. Fedorov M.A., A.F. Grishin A.F. Some questions of the Nevanlinna theory for the complex half-plane // Math. Physics, Analysis and Geometry. 1998. V. 1. № 3. P. 223-271.

8. Ахиезер Н. И. Элементы теории эллиптических функций. М.: Наука, 1970

9. Малютин К. Г. Ряды Фурье и $\delta$-субгармонические функции // Труды ИПММ НАН Украины. 1988. Т. 3. С. 146-157.

10. Левин Б. Я. Распределение корней целых функций. М.: ГИТТЛ, 1956.

11. Хейман У., Кеннеди П. Субгармонические функции. М.: Мир, 1980.

12. Эдвардс Р. Ряды Фурье в современном изложении. Т. 1. М.: Мир, 1985.

13. Эдвардс Р. Ряды Фурье в современном изложении. Т. 2. М.: Мир, 1985.

14. Ронкин Л.И. Регулярность роста и $D^{\prime}$-асимптотика голоморфных функций в $\mathbb{C}^{+} / /$ Изв. вузов. Сер. матем. 1990. № 2. С. 16-28.

15. Говоров Н. В. Краевая задача Римана с бесконечным индексом. М.: Наука, 1986.

Украинская академия банковского дела,

Поступила в редакцию

г. Сумы

13.06.2000

E-mail: malyutin@academia.sumy.ua 